\title{
A Recurrence Plot Based Method for the Detection of End of T-Wave in Abnormal Non-Invasive Fetal Electrocardiogram Signals
}

\author{
Namareq Widatalla ${ }^{1}$, Ahsan Khandoker ${ }^{2}$, Yoshiyuki Kasahara ${ }^{1}$, Yoshitaka Kimura ${ }^{1}$ \\ ${ }^{1}$ Tohoku University, Sendai, Japan \\ ${ }^{2}$ Khalifa University, Abu Dhabi, UAE
}

\begin{abstract}
Features of fetal electrocardiogram (fECG) can convey a lot of information about the function of the heart of the fetus. Up until now, there is little to no research that addresses automatic detection techniques for non-invasive fECG (nfECG). This study discusses a detection technique based on recurrence plots to locate end of T-waves. Identification of the ending timings of T-waves is important to estimate ST and QT intervals. ST and QT intervals are good biomarkers for several cardiovascular diseases that include hypoxia and ischemia. The proposed technique was applied on nfECG records that included normal and abnormal cases. The proposed method could detect end of $T$-waves in most of the analyzed beats. Bland Altman plots of QT and QTC intervals show that at least $95 \%$ of the analyzed beats fall within the limits of agreement (LoA).
\end{abstract}

\section{Introduction}

Fetal heart monitoring during labor is an essential obstetric procedure [1]. Currently, there are many techniques used for monitoring fetal heart function. Two of the mostly used techniques include fetal electrocardiogram (fECG) and Doppler Ultrasound (DUS) [1]. DUS is a non-invasive method which assesses fetal heart rate (fHR) [1]. There are two types of fECG, one is invasive, also known as fetal scalp ECG (fsECG), and the other is non-invasive [2]. FsECG is collected by directly attaching an electrode to the scalp of the fetus. Non-invasive fetal ECG (nfECG) is collected by attaching electrodes to the maternal abdominal surface. After that, an extraction method should be used to separate the maternal ECG (mECG) from the fECG [3]. FsECG is considered accurate compared to DUS and nFECG, however, it can be used only during labor $[4,5]$. In addition, fsECG is considered risky because it can cause infection to the scalp of the fetus [4]. Compared to fsECG, nfECG is considered safer due to its non-invasive nature. In addition, in contrast to fsECG, nfECG's use is not limited to during labor only [1].
To our knowledge, up until this date, little to no research have been addressing automatic detection of nfECG features. Detection techniques developed for nfECG can assist in early diagnosis of fetal heart abnormalities noninvasively. Analysis of nfECG signals is challenging due to their noisy nature [5]. nfECG is usually accompanied with noise due to maternal muscle activity, brain activity of the fetus and movement artifacts [5]. Analyses of QT and ST intervals in nfECG can assist in diagnoses of hypoxia and ischemia $[1,2,6]$.

In order to calculate ST and QT intervals, the ending timings of T-waves should be determined. T-waves are characterized as having low amplitudes and low frequencies. Thus, they get disturbed easily by high frequency noise [7]. In this research, a technique based on recurrence plots is proposed to detect end of T-waves in normal and abnormal nfECG records.

\subsection{Methods}

\subsubsection{Data Collection}

Simultaneous recordings of maternal ECG (mECG), Doppler Ultrasound (DUS), fetal scalp ECG (fsECG) and non-invasive (nfECG) were collected from 7 pregnant women during labor. The signals were collected for a period of 1 minute. 4 of the pregnant women had fetuses with cardiac abnormalities that included heart failure, heart anomaly, tachycardia and umbilical artery dysfunction. The subjects with abnormalities were at gestational age (GA) of 24-34 weeks. Pregnant women bearing healthy fetuses were at GA of 39-41 weeks. The data were collected from Tohoku University Hospital after getting approval from Tohoku University Institutional Review Board, and after getting written informed consent from the subjects.

ECG signals were collected at a sampling rate of 1,000 $\mathrm{Hz}$ and DUS data were collected at $1.15 \mathrm{MHz}$ from Ultrasound Transducer. Doppler recordings were collected to validate the results of the detection. mECG and nfECG signals were recorded by attaching 12-lead on the abdomen 
of the mother. nfECG signals were extracted using blind source separation with reference (BSSR), explained in [8].

\subsubsection{Signal Processing}

nfECG signals were filtered from low and high frequency noise. nfECG signals were decomposed into 10 levels using db4. After that, levels 1- 4 and 7-10 were removed from the signals. Levels $1-4$ had a frequency range of $63-1000 \mathrm{~Hz}$, and levels 7-10 had a frequency range of 1$7 \mathrm{~Hz}$. Not all of the beats within the 1 minute have been analyzed. Beats with high noise and invisible T-waves were removed from the analyses. The total number of beats that have been analyzed was 609 of which 313 were abnormal.

\subsubsection{Recurrence Plots}

Recurrence plot is a technique used for the analyses of dynamical systems. Recurrence plot is mostly used to quantify the recurrences of states or events in a system. Two events are regarded as recurrent if they have similar trajectories [9]. The degree of the similarity between two trajectories is measured by a threshold value. The threshold value is an important parameter that should be chosen properly based on the system. If the threshold value is very large, the number of recurrent events will be very low and vice versa [9].

Since recurrence plots are used to quantity recurrences of events, they can be used to quantity recurrences of events in ECG signals. In this study, recurrence plots have been obtained using MATLAB. The details of the code that was used is explained in $[10,11]$. Since waves in ECG have different amplitudes and characteristics, they can be shown in recurrence plots. Fig. 1 shows a recurrence plot for a $\mathrm{mECG}$ record. As the figure shows, $\mathrm{R}, \mathrm{T}$ and $\mathrm{P}$ peaks are visible in the colored recurrence plots. Since $P$ waves have the smallest amplitudes, they are exhibited in low intensities compared to $\mathrm{R}$ and $\mathrm{T}$ waves. $\mathrm{R}$ peaks have intensities of 0.8 - 1, T-waves have intensities of $0.3-0.6$, and $\mathrm{P}$ waves have intensities below 0.5 . By setting the threshold value to 0.3 , a black and white recurrence plot can be obtained. The black and white plot shows R, T and P waves. Black and white plots have been used to find T-end points in nfECG records.

Compared to mECG, analyses of T-waves in nfECG was more challenging. The reason is due to the more noise presented in nfECG records. Despite applying filters on nfECG records, there was noise that made it hard to distinguish between $\mathrm{T}$ and $\mathrm{P}$ waves. In order to make T-waves distinguishable, the amplitudes of the regions that had Twaves were adjusted. nfECG signals were segmented into beats. The signals were segmented into beats after detecting R-peaks, and separating the regions or intervals that lied between them. After that, the amplitudes of the in- tervals were adjusted using (1), where $x_{a}(t)$ is the adjusted signal and $x(t)$ is the original signal.

$$
x_{a}(t)=2 x(t)+a b s(x(t))
$$

After applying Eq.1, the region that lied within $65 \%$ of length starting from the first $\mathrm{R}$ peak was amplified. A comparison between the recurrence plots of $x(t)$ and the amplified $x_{a}(t)$ of a nfECG record are shown in Fig. 2.
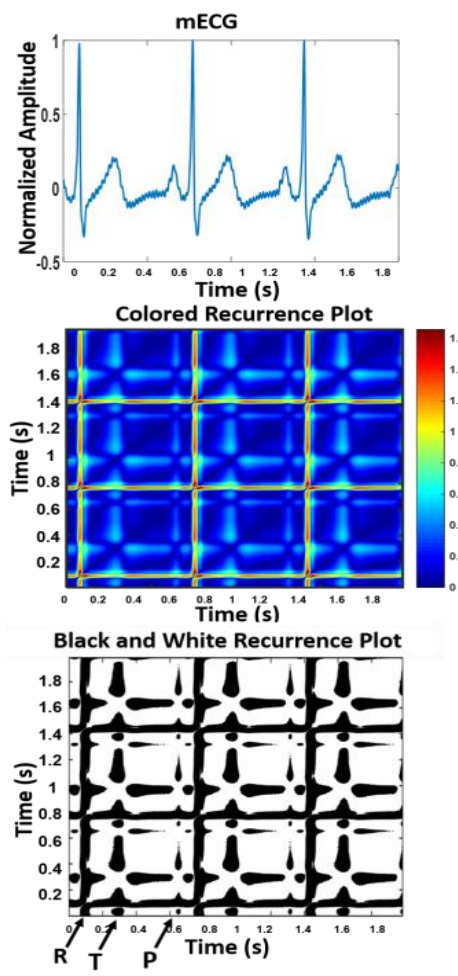

Figure 1. Recurrence plots of mECG.

The obtained recurrence plots could detect the locations of T-waves, however, they could not pinpoint the exact locations of their ends. Thus, the end of a T-wave was determined by finding the minimum value that follows an end of a T-wave of a recurrence plot within the next $0.05 \mathrm{~s}$ in the signal.

\subsubsection{Statistical Analyses}

Detected T-end points were compared with the aortic valve closing timings (Ac) from the Doppler signals [12]. Ac indicates the end of the repolarization phase as explained in [12]. Ac points were recorded manually, after that, Q-Ac and Q-Acc were calculated and compared with QT and QTc of the detected values. QTc and Q-Acc were calculated using Bazzet's formula. Mean and standard deviations of QT and QTc were calculated. In addition, root mean square error (RMSE) was calculated. To test the 


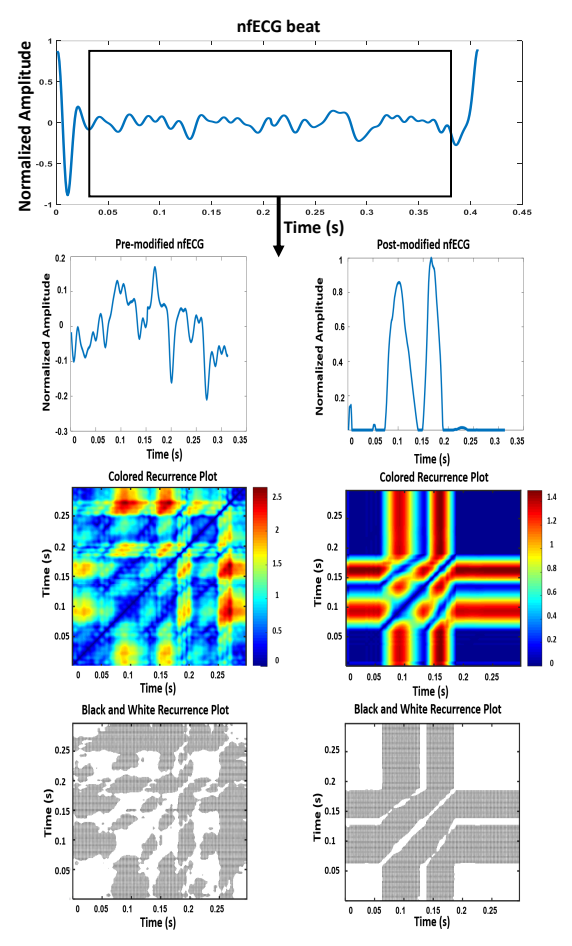

Figure 2. Pre and post modified nfECG.

level of agreement, Bland Altman analysis [13] was performed to count the number of beats that fall within the limits of agreement (LoA).

\section{Results and Discussion}

Figure 3 shows a record of an abnormal nfECG simultaneously compared with a Doppler record. Detected points by the algorithm is simultaneously compared with the Ac timings from the Doppler record. The figure shows that the detected points are close to the Ac timings of the Doppler. In general, detection of end of T-waves in the normal cases was easier than the abnormal cases due to the more noise in the abnormal cases.

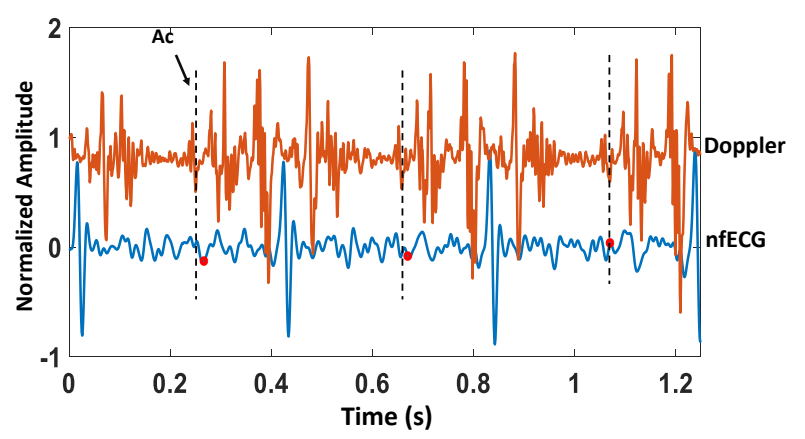

Figure 3. Ac timings compared with detected T-end points
Table 1 shows the mean of QT, QTc, Q-Ac, and Q-Acc for the normal and abnormal cases. As indicated in the table, the difference in mean between detected and actual values is less in the normal case than in the abnormal case. The mean of Q-Ac of normal and abnormal cases is very close, however, the difference in Q-Acc is bigger. The difference in Q-Acc values between normal and abnormal cases is due to the presence of a tachycardia case

Table 2 shows the detailed results of the mean values of QT, Q-Ac, QTc and Q-Acc for the abnormal cases. The detailed results show that the technique was more accurate in cases of heart anomaly and tachycardia. The least accuracy is observed in the placental dysfunction case due to the high noise that affected the visibility of T-waves.

Table 3 shows the results of the RMSE of normal and abnormal cases. The value of RMSE for the normal case is less than the abnormal case. The latter emphasizes the fact that detection of T-waves in normal beats was easier. RMSE results are further validated by the results of the Bland Altman plot summarized in Table 3. The total number of beats within the LoA is larger in the normal case than the abnormal cases. Bland Altman results show that at least $95 \%$ of the analyzed beats fall within the LoA, which shows that the technique was able to detect T-end points in most beats.

Due to the absence of reference values for fetal QT and QTc intervals [6], the results in this study have been compared with previous studies. [14] reports normal values of QT and QTc of $0.234 \mathrm{~s} \pm 0.024 \mathrm{~s}$ and $370 \mathrm{~s} \pm 0.040$ $\mathrm{s}$, respectively (GA: 20-33 weeks). Another research paper, [15], reports normal QT and QTc values of $0.246 \mathrm{~s}$ $\pm 0.028 \mathrm{~s}$ and $0.374 \mathrm{~s} \pm 0.004 \mathrm{~s}$, respectively (GA: 18 41 weeks). The QT value $0.246 \mathrm{~s}$ is close to the normal doppler value of $0.247 \mathrm{~s}$ obtained in this study. The value $0.234 \mathrm{~s}$, reported in [14], is very close to the detected QT value of $0.237 \mathrm{~s}$ for the normal case. The QT and QTc values obtained for the abnormal cases fall outside the normal ranges, $0.234 \mathrm{~s}-0.246 \mathrm{~s}$ and $0.370 \mathrm{~s}-0.374 \mathrm{~s}$, mentioned in the studies, $[14,15]$.

\section{Conclusion}

This study discussed a method based on recurrence plots for detecting end of T-waves in normal and abnormal nfECG signals. The results of Bland Altman showed that the technique was effective in evaluating T-end points. Detection of T-waves in normal cases was easier compared to abnormal cases due to the less noise in the normal case. Although the results obtained in this study shows that recurrence plots could detect T-waves in nfECG records, more challenging nfECG records should be analyzed for validation. 
Table 1. Comparison between actual and detected QT,Q-Ac and QTc,(Q-Ac)c in normal and abnormal cases.

\begin{tabular}{|c|c|c|c|c|}
\hline Feature & \multicolumn{2}{|c|}{ Normal } & \multicolumn{2}{|c|}{ Abnormal } \\
\hline & Doppler & Detected & Doppler & De \\
\hline Num & \multicolumn{2}{|c|}{296} & \multicolumn{2}{|c|}{31} \\
\hline QT or Q-Ac (s) & 0.013 & $0.237 \pm 0.0$ & $0.244 \pm 0.031$ & $0.228 \pm 0.044$ \\
\hline QTc or Q-Acc (s) & $0.368 \pm 0.019$ & $0.352 \pm 0.045$ & $0.392 \pm 0.037$ & $0.367 \pm 0.067$ \\
\hline
\end{tabular}

Table 2. Comparison between actual and detected QT,Q-Ac and QTc,(Q-Ac)c in abnormal cases.

\begin{tabular}{lccccccccr}
\hline \hline Feature & \multicolumn{2}{c}{ Heart Anomaly } & \multicolumn{2}{c}{ Heart Failure } & Placental Dysfunction & \multicolumn{2}{c}{ Tachycardia } \\
\hline & Doppler & Detected & Doppler & Detected & Doppler & Detected & Doppler & Detected \\
\hline Number of beats & \multicolumn{2}{c}{97} & \multicolumn{2}{c}{74} & \multicolumn{2}{c}{96} & \multicolumn{2}{c}{46} \\
QT or Q-Ac (s) & $0.259 \pm$ & $0.258 \pm$ & $0.25 \pm$ & $0.232 \pm$ & $0.252 \pm$ & $0.212 \pm$ & $0.185 \pm$ & $0.190 \pm$ \\
& 0.019 & 0.039 & 0.019 & 0.046 & 0.014 & 0.032 & 0.026 & 0.028 \\
QTc or Q-Acc (s) & $0.41 \pm$ & $0.41 \pm$ & $0.389 \pm$ & $0.362 \pm$ & $0.396 \pm$ & $0.333 \pm$ & $0.350 \pm$ & $0.357 \pm$ \\
& 0.029 & 0.062 & 0.03 & 0.073 & 0.022 & 0.05 & 0.049 & 0.052
\end{tabular}

Table 3. Results of RMSE and Bland Altman.

\begin{tabular}{lrr}
\hline \hline Feature & Normal & Abnormal \\
\hline \multicolumn{3}{c}{ RMSE } \\
\hline QT or Q-Ac (s) & 0.0359 & 0.0447 \\
QTc or Q-Acc (s) & 0.0536 & 0.0713 \\
\hline \multicolumn{2}{c}{ Bland Altman } \\
\hline QT or Q-Ac (s) & $97 \%$ & $95 \%$ \\
QTc or Q-Acc (s) & $97 \%$ & $95 \%$
\end{tabular}

\section{Acknowledgments}

The work in this paper has been supported by RIKEN Health-care and Medical Data Platform Project.

\section{References}

[1] W. Adam, "The future of fetal monitoring," Rev Obstet Gynecol., vol. 5, no. 3-4, pp. 132-136, 2012.

[2] G. Clifford, I. Silva, J. Behar and G. Moody, "Noninvasive fetal ECG analysis," Physiol Meas., vol. 35, no. 8, p. 1521-1536, 2014.

[3] A. Khandoker, E. Ibrahim, S. Oshio and Y. Kimura, "Validation of beat by beat fetal heart signals acquired from four-channel fetal phonocardiogram with fetal electrocardiogram in healthy late pregnancy," Scientific Reports, vol. 8, no. 1, pp. 1-11, 2018.

[4] L. Su and H.-T. Wu, "Extract fetal ECG from single-lead abdominal ECG by de-shape short time Fourier transform and nonlocal median," Front. Appl. Math. Stat., vol. 3, 2017.

[5] R. Sameni and G. Clifford, "A review of fetal ECG signal processing; issues and promising directions," Open Pacing Electrophysiol Ther J., vol. 3, no. 1, pp. 4-20.

[6] J. Behar, T. Z. Zhu, J. Oster, A. Niksch, D. Mah, T. Chun, et al., "Evaluation of the fetal QT interval using non-invasive fetal ECG technology," Physiol Meas. , vol. 37, no. 9, p. 1392-1403, 2016.

[7] M. Elgendi, B. Eskofier and D. Abott, "Fast T wave detection calibrated by clinical knowledge with annotation of $\mathrm{P}$ and $\mathrm{T}$ waves," Sensors, vol. 15, no. 7, p. 17693-17714, 2015.

[8] M. Sato, Y. Kimura, S. Chida, T. Ito, N. Katayama, K. Okamura, et al., "A novel extraction method of fetal electrocardiogram from the composite abdominal signal," IEEE Transactions on Biomedical Engineering, vol. 54, no. 1, pp. 49 - 58, 2007.
[9] N. Marwan, M. C. Romano, M. Thiel and J. Kurths, "Recurrence plots for the analysis of complex systems," Physics Reports, vol. 438, p. $237-329,2007$.

[10] H. Yang, "Multiscale recurrence quantification analysis of spatial cardiac vectorcardiogram signals," IEEE T BIO-MED ENG, vol. 58, no. 2, pp. 339-347, 2010.

[11] Y. Chen and H. Yang, "Multiscale recurrence analysis of long-term nonlinear and nonstationary time series," Chaos, Solitons \& Fractals, vol. 45, no. 7, pp. 978-987, 2012.

[12] A. Khandoker, Y. Kimura, T. Ito, N. Sato, K. Okamura and M. Palaniswami, "Antepartum non-invasive evaluation of opening and closing timings of the cardiac valves in fetal cardiac cycle," Med Biol Eng Comput., vol. 47, no. 10, p. 1075-1082, 2009.

[13] J. Bland and D. Altman, "Measuring agreement in method comparison studies," Stat Methods Med Res., vol. 8, no. 2, p. 135-160, 1999.

[14] C. Velayo, K. Funamoto, J. Silao, Y. Kimura and K. Nicolaides, "Evaluation of abdominal fetal electrocardiography in early intrauterine growth restriction," Front Physiol, vol. 8, no. 837, 2017.

[15] N. Sato, T. Hoshiai, T. Ito, K. Owada, H. Chisaka, A. Aoyagi, et al., "Successful detection of the fetal electrocardiogram waveform changes during various states of singletons," Tohoku J Exp Med., vol. 225, no. 2, pp. 89-94, 2011.

Address for correspondence:

Namareq Widatalla

980-8575

Kimura Laboratory

Tohoku University School of Medicine

9th Floor of building No.5

2-1 Seiryo-machi, Aoba-ku, Sendai, Miyagi

JAPAN

namareq.widatalla@gmail.com 